\title{
Schwarzschild Geodesics in Terms of Elliptic Functions and the Related Red Shift
}

\author{
Günter Scharf \\ Institut für Theoretische Physik, Universität Zürich, Switzerland \\ E-mail:scharf@physik.unizh.ch \\ Received February 5, 2010; revised March 10, 2011; accepted March 15, 2011
}

\begin{abstract}
Using Weierstrassian elliptic functions the exact geodesics in the Schwarzschild metric are expressed in a simple and most transparent form. The results are useful for analytical and numerical applications. For example we calculate the perihelion precession and the light deflection in the post-Einsteinian approximation. The bounded orbits are computed in the post-Newtonian order. As a topical application we calculate the gravitational red shift for a star moving in the Schwarzschild field.
\end{abstract}

Keywords: Schwarzschild Geodesics, Red Shift

\section{Introduction}

Schwarzschild geodesics are elliptic functions, therefore, they should be written as such. For this purpose the Weierstrassian elliptic functions are most useful because they lead to simple expressions. The reason for this is that the solution of quartic or cubic equations can be avoided in this way.

In a recent paper [1] an analytic solution for the geodesic in the weak-field approximation was given. As pointed out in that paper the progress in the astronomical observations call for better analytical methods. In this respect it is desirable to have the exact geodesics in a form most suited for applications. For the orbits in polar coordinates (next section) this goal can be achieved by using Weierstrass' $P$-function for which many analytical and numerical methods are known [2]. Considering the motion in time (section 4) the related $\zeta$ - and $\sigma$-functions of Weierstrass appear.

Jacobian elliptic functions have been used by Darwin [8] for the form of the orbits. After some transformation our result (2.13) agrees with his. But in his second paper he abandons the elliptic functions because they were "not so well adapted to a study of the time in those orbits". Obviously the Weierstrass functions are better suited for the problem. Indeed, expressing them by Theta functions one gets the natural expansion of the geodesics in powers of the Schwarzschild radius, this expansion involves elementary functions only. The Weierstrass functions have also been used by Hagihara $[11]^{1}$. But he has chosen the variables and constants of integration in a manner which leads to less explicit results. So it is difficult to derive the post-Newtonian corrections to the geodesics given here from his formulas. As a topical application we finally calculate the red shift for a star moving in the Schwarzschild field. The geodesics are also needed for the study of modifications of general relativity ([10], section 5.13).

\section{The Orbits in Polar Coordinates $r=r(\varphi)$}

We take the coordinates $x^{0}=c t, x^{1}=r, x^{2}=\vartheta$, $x^{3}=\varphi$ and write the Schwarzschild metric in the form

$\mathrm{d} s^{2}=\frac{r-r_{s}}{r} c^{2} \mathrm{~d} t^{2}-\frac{r}{r-r_{s}} \mathrm{~d} r^{2}-r^{2}\left(\mathrm{~d} \vartheta^{2}+\sin ^{2} \vartheta \mathrm{d} \varphi^{2}\right)$

where $r_{s}=\frac{2 G M}{c^{2}}$ is the Schwarzschild radius. We shall assume $c=1$ in the following. The geodesic equation

$$
\frac{\mathrm{d}^{2} x^{\alpha}}{\mathrm{d} s^{2}}+\Gamma_{\beta \gamma}^{\alpha} \frac{\mathrm{d} x^{\beta}}{\mathrm{d} s} \frac{\mathrm{d} x^{\gamma}}{\mathrm{d} s}=0
$$

with the Christoffel $\Gamma_{\beta \gamma}^{\alpha}$ leads to the following three differential equations

${ }^{1}$ I am indebted to C. Lämmerzahl and P. Fiziev for bringing this reference to my attention 


$$
\begin{gathered}
\frac{\mathrm{d}^{2} t}{\mathrm{~d} s^{2}}+v^{\prime} \frac{\mathrm{d} t}{\mathrm{~d} s} \frac{\mathrm{d} r}{\mathrm{~d} s}=0 \\
\frac{\mathrm{d}^{2} r}{\mathrm{~d} s^{2}}+\frac{v^{\prime}}{2} e^{2 v}\left(\frac{\mathrm{d} t}{\mathrm{~d} s}\right)^{2}-\frac{v^{\prime}}{2}\left(\frac{\mathrm{d} r}{\mathrm{~d} s}\right)^{2}-r e^{v}\left(\frac{\mathrm{d} \varphi}{\mathrm{d} s}\right)^{2}=0 \\
\frac{\mathrm{d}^{2} \varphi}{\mathrm{d} s^{2}}+\frac{2}{r} \frac{\mathrm{d} r}{\mathrm{~d} s} \frac{\mathrm{d} \varphi}{\mathrm{d} s}=0
\end{gathered}
$$

Here we have used the standard representation

$$
1-\frac{r_{s}}{r}=e^{v}
$$

and have chosen $\vartheta=\pi / 2$ as the plain of motion. The Christoffel symbols can be taken from the Appendix of [3].

Multiplying (2.3) by $\exp v$ we find

$$
\frac{\partial}{\mathrm{d} s}\left(e^{v} \frac{\mathrm{d} t}{\mathrm{~d} s}\right)=0
$$

so that

$$
\begin{gathered}
e^{v} \frac{\mathrm{d} t}{\mathrm{~d} s}=\text { const. }=E \\
\frac{\mathrm{d} t}{\mathrm{~d} s}=E e^{-v} .
\end{gathered}
$$

Next multiplying (2.5) by $r^{2}$ we get

$$
r^{2} \frac{\mathrm{d} \varphi}{\mathrm{d} s}=\text { const. }=L
$$

hence

$$
\frac{\mathrm{d} \varphi}{\mathrm{d} s}=\frac{L}{r^{2}} .
$$

For the constants of integration we use the notation of Chandrasekhar [4].

Finally, substituting (2.7) and (2.8) into (2.5) and multiplying by $2 \exp (-v) \times \mathrm{d} r / \mathrm{d} s$ we obtain

$$
\frac{\mathrm{d}}{\mathrm{d} s}\left[e^{-v}\left(\frac{\mathrm{d} r}{\mathrm{~d} s}\right)^{2}-E^{2} e^{-v}+\frac{L^{2}}{r^{2}}\right]=0 .
$$

Consequently, the square bracket is equal to another constant $=b$. Then the resulting differential equation can be written as

$$
\left(\frac{\mathrm{d} r}{\mathrm{~d} s}\right)^{2}=E^{2}+e^{v}\left(b-\frac{L^{2}}{r^{2}}\right)
$$

The constant $b$ can be arbitrarily adjusted by rescaling the affine parameter $s$. Below we shall take $b=-m^{2}$ where $m$ is the rest mass of the test particle. This will enable us to include null geodesics (light rays) with $m^{2}=0$. Each geodesic is characterized by two constants of the motion: energy $E$ and angular momentum $L$.
Taking the square root of (2.10) and dividing by (2.8) we get

$$
\frac{\mathrm{d} r}{\mathrm{~d} \varphi}=\sqrt{\frac{E^{2}-m^{2}}{L^{2}} r^{4}+\frac{m^{2}}{L^{2}} r_{s} r^{3}-r^{2}+r_{s} r} \equiv \sqrt{f(r)} .
$$

Now $\varphi=\varphi(r)$ can be written as an elliptic integral. However, it is better to consider the inverse $r=r(\varphi)$ in terms of elliptic function by using a formula of Weierstrass ([5], p.452). Let the quartic $f(r)$ be written as

$$
f(r)=a_{0} r^{4}+4 a_{1} r^{3}+6 a_{2} r^{2}+4 a_{3} r+a_{4},
$$

and let $r_{1}$ be a zero $f\left(r_{1}\right)=0$, then a solution of (2.11) is given by

$$
r=r_{1}+\frac{f^{\prime}\left(r_{1}\right)}{4 P\left(\varphi ; g_{2}, g_{3}\right)-f^{\prime \prime}\left(r_{1}\right) / 6} .
$$

Here $P\left(\varphi ; g_{2}, g_{3}\right)$ is Weierstrass' $P$-function with invariants

$$
\begin{gathered}
g_{2}=a_{0} a_{4}-4 a_{1} a_{3}+3 a_{2}^{2} \\
g_{3}=a_{0} a_{2} a_{4}+2 a_{1} a_{2} a_{3}-a_{2}^{3}-a_{0} a_{3}^{2}-a_{1}^{2} a_{4} .
\end{gathered}
$$

In our case we have $a_{4}=0$. For the convenience of the reader we reproduce the short proof in the Appendix.

The result (2.13) is not yet the solution of our problem because it contains too many constants: the invariants $g_{2}, g_{3}$ and the derivatives of $f$ depend on $E, L$, but in addition the zero $r_{1}$ appears. Of course one could calculate $r_{1}$ as a function of $E, L$ by solving the quartic equation $f(r)=0$, but this gives complicated expressions. It is much better to use $r_{1}$ and a second zero $r_{2}$ as constants of integration instead of $E, L$. This is even desirable from the astronomers point of view because the zeros of derivative (2.11) are turning points of the geodesic, for example in case of a bounded orbit they can be identified with the perihelion and aphelion of the orbit. In order to express $E, L$ by $r_{1}, r_{2}$ we write our quartic in the form

$$
f(r)=a_{0} r\left(r-r_{1}\right)\left(r-r_{2}\right)\left(r-r_{3}\right)
$$

and compare the coefficients of $r^{3}, r^{2}, r$ with (2.12). This leads to

$$
\begin{gathered}
4 a_{1}=-a_{0}\left(r_{1}+r_{2}+r_{3}\right)=\frac{m^{2}}{L^{2}} r_{s} \\
6 a_{2}=a_{0}\left(r_{1} r_{2}+r_{1} r_{3}+r_{2} r_{3}\right)=-1 \\
4 a_{3}=-a_{0} r_{1} r_{2} r_{3}=r_{s} .
\end{gathered}
$$

Since

$$
a_{0}=\frac{E^{2}-m^{2}}{L^{2}}
$$

we can solve for 


$$
\begin{aligned}
& \frac{m^{2}}{L^{2}} r_{s}=\frac{r_{1}+r_{2}+r_{3}}{r_{1} r_{2}+r_{1} r_{3}+r_{2} r_{3}}, \\
& E^{2}-m^{2}=-\frac{m^{2} r_{s}}{r_{1}+r_{2}+r_{3}}
\end{aligned}
$$

In addition we obtain the third zero

$$
r_{3}=r_{s} \frac{r_{1} r_{2}}{r_{1} r_{2}-r_{1} r_{s}-r_{2} r_{s}} .
$$

The relations (2.19-21) allow to express everything in terms of $r_{1}, r_{2}$. For the invariants we find

$$
\begin{aligned}
& g_{2}=\frac{1}{12}-\frac{m^{2}}{4 L^{2}} r_{s}^{2}=\frac{1}{12}-\frac{r_{s}}{4} \frac{r_{1}+r_{2}+r_{3}}{r_{1} r_{2}+r_{1} r_{3}+r_{2} r_{3}} \\
g_{3}= & \frac{1}{6^{3}}-\frac{m^{2}}{48 L^{2}} r_{s}^{2}-\frac{a_{0}}{16} r_{s}^{2} \\
= & \frac{1}{6^{3}}-\frac{1}{48} \frac{\left(r_{1}+r_{2}+r_{3}\right) r_{s}}{r_{1} r_{2}+r_{1} r_{3}+r_{2} r_{3}}+\frac{1}{16} \frac{r_{s}^{2}}{r_{1} r_{2}+r_{1} r_{3}+r_{2} r_{3}} .
\end{aligned}
$$

Here $r_{3}$ has to be substituted by (2.21). For the derivatives $f^{\prime}\left(r_{1}\right), f^{\prime \prime}\left(r_{1}\right)$ which appear in our solution (2.13) we obtain

$$
\begin{gathered}
f^{\prime}\left(r_{1}\right)=\frac{-r_{1}\left(r_{1}-r_{2}\right)\left(r_{1}-r_{3}\right)}{r_{1} r_{2}+r_{1} r_{3}+r_{2} r_{3}} \\
f^{\prime \prime}\left(r_{1}\right)=-2 \frac{\left(r_{1}-r_{2}\right)\left(r_{1}-r_{3}\right) r_{1}+\left(r_{1}-r_{2}\right)\left(r_{1}-r_{3}\right)}{r_{1} r_{2}+r_{1} r_{3}+r_{2} r_{3}} .
\end{gathered}
$$

With these substitutions the result (2.13) gives all possible geodesics in the form $r=r\left(\varphi ; r_{1}, r_{2}\right)$. This will be discussed in the next section.

As a first check of the solution (2.13) we consider the Newtonian limit. Let the two zeros $r_{1}, r_{2}$ be real and large compared to the Schwarzschild radius $r_{s}$ in absolute value. Then neglecting $O\left(r_{s}\right)$ in the invariants (2.22-23) the $P$-function becomes elementary ([2], p.652, equation 18.12.27):

$$
P\left(\varphi ; \frac{1}{12}, 6^{-3}\right)=-\frac{1}{12}+\frac{1}{4 \sin ^{2} \varphi / 2}=-\frac{1}{12}+\frac{1}{2(1-\cos \varphi)} .
$$

The leading order in the derivatives of $f$ is given by

$$
\begin{gathered}
f^{\prime}\left(r_{1}\right)=-\frac{r_{1}}{r_{2}}\left(r_{1}-r_{2}\right) \\
f^{\prime \prime}\left(r_{1}\right)=-2\left(3 \frac{r_{1}}{r_{2}}-2\right) .
\end{gathered}
$$

It is convenient to introduce the eccentricity $\varepsilon$ by

$$
\frac{r_{1}}{r_{2}}=\frac{1-\varepsilon}{1+\varepsilon} \text {. }
$$

Using all this in (2.13) we find the wellknown conic

$$
r=\frac{(1+\varepsilon) r_{1}}{1+\varepsilon \cos \varphi}
$$

Assuming both zeros $r_{1}, r_{2}$ positive and $r_{1}<r_{2}$ we have $\varepsilon<1$ and the orbit is an ellipse with perihelion $r_{1}$ and aphelion $r_{2}$. In the hyperbolic case $\varepsilon>1$ we see from (2.28) that if $r_{1}$ is positive $r_{2}$ must be negative. Then there is only one physical turning point $r_{1}$ which is the point of closest approach. The latter always corresponds to $\varphi=0$. The relativistic corrections to (2.29) are calculated in the following section.

\section{Discussion of the Solution}

The solution $r=r(\varphi)$ (2.13) is an elliptic function of $\varphi$ which implies that it is doubly-periodic ([2], p.629 or any book on elliptic functions). The values of the two half-periods $\omega, \omega^{\prime}$ depend on the three roots of the fundamental cubic equation

$$
4 e^{3}-g_{2} e-g_{3}=0 \text {. }
$$

Again it is not necessary to solve this equation because the solutions $e_{j}, j=1,2,3$ can be easily obtained from the roots $0, r_{1}, r_{2}, r_{3}$ of our quartic $f(r)=0$. To see this we transform $f(r)$ to Weierstrass' normal form as follows. First we set $r=1 / x$ so that from (2.16) we get

$$
f(r)=\frac{1}{x^{4}}\left(4 a_{3} x^{3}+6 a_{2} x^{2}+4 a_{1} x+a_{0}\right)
$$

Next we remove the quadratic term by introducing

$$
\frac{1}{r}=x=\frac{1}{a_{3}}\left(e-\frac{a_{2}}{2}\right) \text {. }
$$

This gives the normal form of Weierstrass

$$
f(r)=\frac{a_{3}^{2}}{\left(e-12 a_{2}\right)^{4}}\left(4 e^{3}-g_{2} e-g_{3}\right),
$$

with the above invariants (2.14-15). That means roots of $f(r)$ are simply related to roots of (3.1) by the transformation

$$
e_{j}=\frac{a_{3}}{r_{j}}+\frac{a_{2}}{2}=\frac{r_{s}}{4 r_{j}}-\frac{1}{12} .
$$

The cubic equation (3.1) with real coefficients has either three real roots or one real and two complex conjugated roots. The first case occurs if the discriminant

$$
\Delta=g_{2}^{3}-27 g_{3}^{2}
$$

is positive, in the second case $\Delta$ is negative. In terms of the roots $\Delta$ is given by ([2], p.629, equation 18.1.8)

$$
\Delta=16\left(e_{1}-e_{2}\right)^{2}\left(e_{2}-e_{3}\right)^{2}\left(e_{3}-e_{1}\right)^{2} \text {. }
$$


The physically interesting orbits correspond to the first case of real roots. If we have two complex conjugated zeros $r_{2}=r_{1}^{*}$ then (2.28) implies that the eccentricity $\varepsilon$ is imaginary. Such orbits have been discussed by Chandrasekhar ([4], p.111). Now we discuss the various cases.

\subsection{Bound Orbits}

In this case we have two positive turning points $r_{2}>r_{1}>0$, consequently there are three real roots $e_{1}>0>e_{2}>e_{3}$ given by

$$
\begin{gathered}
e_{1}=\frac{r_{s}}{4 r_{3}}-\frac{1}{12}=\frac{1}{6}-\frac{r_{s}}{4} \frac{r_{1}+r_{2}}{r_{1} r_{2}} \\
e_{2}=-\frac{1}{12}+\frac{r_{s}}{4 r_{1}}, \quad e_{3}=-\frac{1}{12}+\frac{r_{s}}{4 r_{2}},
\end{gathered}
$$

Our convention is chosen in agreement with [2]. The real half-period $\omega$ of the $P$-function is given by ([2], p.549, equation 18.9.8)

$$
\begin{aligned}
& \omega=\int_{e_{1}}^{\infty} \frac{\mathrm{d} t}{\sqrt{4 t^{3}-g_{2} t-g_{3}}}=\frac{K\left(k^{2}\right)}{\sqrt{e_{1}-e_{3}}} \\
& \omega=\pi\left\{1+\frac{3 r_{s}}{4} \frac{r_{1}+r_{2}}{r_{1} r_{2}}+\frac{3 r_{s}^{2}}{8\left(r_{1} r_{2}\right)^{2}}\left[\left(2 r_{1}+r_{2}\right)\left(2 r_{2}+r_{1}\right)+\frac{3}{8}\left(r_{2}-r_{1}\right)^{2}\right]\right\}=\pi\left\{1+\frac{3}{2} \frac{r_{s}}{r_{1}} \frac{1}{1+\varepsilon}+\frac{3}{18} \frac{r_{s}^{2}}{r_{1}^{2}} \frac{18+\varepsilon^{2}}{(1+\varepsilon)^{2}}+O\left(r_{s}^{3}\right)\right\}
\end{aligned}
$$

The perihelion precession is given by $\Delta \varphi=2(\omega-\pi)$. Then the order $r_{s}$ in (3.12) is Einstein's result and the $O\left(r_{s}^{2}\right)$ gives the correction to it. The accurate computation of the half-period is necessary to control the orbit in the large.

To compute the relativistic corrections for $r(\varphi)$ from (2.13) we express the $P$-function by Theta functions ([5], p.464)

$$
\begin{aligned}
& \vartheta_{1}(z, q)=2 q^{1 / 4}\left(\sin z-q^{2} \sin 3 z+q^{6} \sin 5 z-\cdots\right) \\
& \vartheta_{2}(z, q)=2 q^{1 / 4}\left(\cos z+q^{2} \cos 3 z+q^{6} \cos 5 z+\cdots\right) \\
& \vartheta_{3}(z, q)=1+2 q\left(\cos 2 z+q^{3} \cos 4 z+q^{8} \cos 6 z+\cdots\right) \\
& \vartheta_{4}(z, q)=1-2 q\left(\cos 2 z-q^{3} \cos 4 z+q^{8} \cos 6 z-\cdots\right) .
\end{aligned}
$$

Here $q$ is the so-called Nome ([2], eq. 17.3.21)

$$
q=\frac{k^{2}}{16}+8\left(\frac{k^{2}}{16}\right)^{2}+\cdots
$$

These series are rapidly converging since $k^{2}$ is small where $K\left(k^{2}\right)$ is the complete elliptic integral of the first kind with parameter

$$
\begin{aligned}
k^{2} & =\frac{e_{2}-e_{3}}{e_{1}-e_{3}}=r_{s} \frac{r_{2}-r_{1}}{r_{1} r_{2}-r_{s}\left(2 r_{1}+r_{2}\right)} \\
& =\frac{r_{s}}{r_{1}} \frac{2 \varepsilon}{1+\varepsilon}\left(1-r_{s} \frac{3-\varepsilon}{1+\varepsilon}\right)^{-1} .
\end{aligned}
$$

As a first application let us give the post-Einsteinian correction to the orbital precession. If $k^{2}$ (3.10) is small we can use the expansion ([2], p.591, equation 17.3.11)

$$
K\left(k^{2}\right)=\frac{\pi}{2}\left[1+\left(\frac{1}{2}\right)^{2} k^{2}+\left(\frac{1 \cdot 3}{2 \cdot 4}\right)^{2} k^{4}+\cdots\right]
$$

From the roots $e_{1}, e_{3}$ we find

$$
\frac{1}{\sqrt{e_{1}-e_{3}}}=2\left[1+\frac{r_{s}}{2} \frac{2 r_{1}+r_{2}}{r_{1} r_{2}}+\frac{3}{8} r_{s}^{2}\left(\frac{2 r_{1}+r_{2}}{r_{1} r_{2}}\right)^{2}+O\left(r_{s}^{3}\right)\right] \text {. }
$$

(3.10), they give the natural expansion in powers of the $S c h w a r z s c h i l d$ radius $r_{s}$. Now the $P$-function is given in terms of Theta functions by ([2], eq. 18.10.5)

$$
\begin{aligned}
& P(\varphi)=e_{2}+\frac{\pi^{2}}{4 \omega^{2}}\left(\frac{\vartheta_{1}^{\prime}(0)}{\vartheta_{3}(0)} \frac{\vartheta_{3}(\phi)}{\vartheta_{1}(\phi)}\right)^{2} \\
& \quad=-\frac{1}{12}+\frac{r_{s}}{4 r_{1}}+\frac{\pi^{2}}{4 \omega^{2}} \frac{1}{\sin ^{2} \phi}\left[1+4 q\left(\cos ^{2} \phi-1\right)+O\left(q^{2}\right)\right],
\end{aligned}
$$

where

$$
\phi=\frac{\pi}{2 \omega} \varphi .
$$

Using

$$
\begin{aligned}
& f^{\prime}\left(r_{1}\right)=2 r_{1}\left(\frac{\varepsilon}{1+\varepsilon}-\frac{r_{s}}{r_{1}} \frac{3 \varepsilon+\varepsilon^{2}}{(1+\varepsilon)^{2}}\right) \\
& f^{\prime \prime}\left(r_{1}\right)=-2 \frac{1-5 \varepsilon}{1+\varepsilon}+6 \frac{r_{s}}{r_{1}} \frac{1-4 \varepsilon-\varepsilon^{2}}{(1+\varepsilon)^{2}}
\end{aligned}
$$

this leads to

$$
P(\varphi)-\frac{f^{\prime \prime}\left(r_{1}\right)}{24}=\frac{1+\varepsilon-2 \varepsilon \sin ^{2} \phi}{4(1+\varepsilon) \sin ^{2} \phi}\left[1+\frac{r_{s}}{r_{1}} \frac{1}{1+\varepsilon \cos 2 \phi}\left(-3-\frac{\varepsilon}{2}(1-\cos \phi)++2 \varepsilon \frac{3+\varepsilon}{1+\varepsilon} \sin ^{2} \phi\right)\right]+O\left(\frac{r_{s}}{r_{1}}\right)^{2} .
$$


Substituting this into (2.13) gives the desired orbit to $O\left(r_{s}\right)$

$$
\frac{r(\varphi)}{r_{1}}=\frac{1+\varepsilon}{1+\varepsilon \cos 2 \phi}+\frac{r_{s}}{r_{1}} \varepsilon \frac{2 \sin ^{2} \phi}{1+\varepsilon \cos 2 \phi}\left\{\frac{1}{1+\varepsilon \cos 2 \phi}\left[3+\frac{\varepsilon}{2}(1-\cos \phi)-2 \varepsilon \frac{3+\varepsilon}{1+\varepsilon} \sin ^{2} \phi\right]-\frac{3+\varepsilon}{1+\varepsilon}\right\} .
$$

It is important to insert the period $\omega$ in $\phi$ (3.16) according to (3.12) in order to describe the perihelion precession correctly.

If the two roots $r_{1}=r_{2}$ coincide, it follows from (2.24) that $f^{\prime}\left(r_{1}\right)=0$. According to (2.13) we then have circular motion $r=r_{1}$. If all three zeros coincide $r_{1}=r_{2}=r_{3}$ then (2.21) gives $r_{3}=3 r_{s}$ which is the innermost circular orbit.

\subsection{Unbound Orbits}

In this case there is only one physical point, the point of closest approach $r_{1}$. The other root $r_{2}$ is negative, therefore, it is better to use the eccentricity $\varepsilon$ (2.27) as the second basic quantity. With $r_{3}$ given by (2.21) we then have

$$
r_{1}>r_{3}>0>r_{2}=\frac{1+\varepsilon}{1-\varepsilon} r_{1},
$$

because $\varepsilon \geq 1$. The periodicity of (2.13) in $\varphi$ is now realized by a jump to an unphysical branch with $r<0$. In reality a comet moves on one branch only, but it is a tricky problem to decide on which one. This is due to the fact that the period differs a little from $2 \pi$ as in the bounded case. Consequently, neighboring physical branches $r>0$ are a little rotated against each other and the distinction between them is not easy. The quantity of physical interest is the direction $\varphi_{\infty}$ of the asymptote. It follows from the original equation (2.11) by integrating the inverse over $r$ from $r_{1}$ to $\infty$

$$
\varphi_{\infty}=\int_{n}^{\infty} \frac{\mathrm{d} r}{\sqrt{f(r)}} .
$$

This is an elliptic integral which can be transformed to Legendre's normal form

$$
\varphi_{\infty}=\frac{\mu}{\sqrt{a_{0}}} \int_{0}^{\Phi_{2}} \frac{\mathrm{d} \Phi}{\sqrt{1-k^{2} \sin ^{2} \phi}}
$$

by the transformation ([6], vol.II, p.308)

$$
\sin ^{2} \Phi=\frac{r_{3}-r_{2}}{r_{1}-r_{2}} \frac{r-r_{1}}{r-r_{3}}, \quad \sin ^{2} \Phi_{2}=\frac{r_{3}-r_{2}}{r_{1}-r_{2}} .
$$

The parameter $k^{2}$ in (3.22) is given by

$$
k^{2}=\frac{r_{3}}{r_{1}} \frac{r_{1}-r_{2}}{r_{3}-r_{2}}
$$

and

$$
\mu=\frac{2}{\sqrt{r_{1}\left(r_{3}-r_{2}\right)}} .
$$

The integral (3.22) is an incomplete elliptic integral of the first kind

$$
\varphi_{\infty}=\frac{\mu}{\sqrt{a_{0}}} F\left(\Phi_{2}, k^{2}\right)
$$

which has the expansion ([6], vol.II, p.313)

$$
F\left(\Phi_{2}, k^{2}\right)=\Phi_{2}+\frac{k^{2}}{4}\left(\Phi_{2}-\frac{1}{2} \sin 2 \Phi_{2}\right)+O\left(k^{4}\right) .
$$

For small $r_{s} / r_{1}$ we find

$$
\begin{gathered}
k^{2}=\frac{2 \varepsilon}{\varepsilon+1} \frac{r_{s}}{r_{1}}+O\left(r_{s} r_{1}\right)^{2} \\
\frac{\mu}{\sqrt{a_{0}}}=2+\frac{3-\varepsilon}{1+\varepsilon} \frac{r_{s}}{r_{1}}+O\left(r_{s} r_{1}\right)^{2} \\
\sin ^{2} \Phi_{2}=\frac{\varepsilon+1}{2 \varepsilon}\left(1-\frac{r_{3}}{r_{1}}\right) .
\end{gathered}
$$

This gives

$$
\cos 2 \Phi_{2}=-\frac{1}{\varepsilon}-\frac{\varepsilon-1}{\varepsilon} \frac{r_{3}}{r_{1}}
$$

and

$$
\varphi_{\infty}=2 \Phi_{2}+\frac{r_{s}}{r_{1}}\left(\frac{3}{\varepsilon+1} \Phi_{2}-\frac{\varepsilon}{2 \varepsilon+2} \sin 2 \Phi_{2}\right) .
$$

It is convenient to calculate

$$
\cos \varphi_{\infty}=-\frac{1}{\varepsilon}-\frac{r_{s}}{r_{1}}\left(\frac{\varepsilon-1}{2 \varepsilon}+3 \frac{\sqrt{\varepsilon^{2}-1}}{\varepsilon(\varepsilon+1)} \Phi_{2}\right)+O\left(r_{s}^{2}\right) .
$$

The leading order is the Newtonian asymptote of the hyperbola.

\subsection{Null Geodesics}

For $m^{2}=0$ there is only one constant of integration in the quartic (2.11)

$$
f(r)=\frac{r^{4}}{d^{2}}-r^{2}+r_{s} r
$$

which is the so-called impact parameter

$$
d=\frac{L}{E} .
$$


Now it is necessary to calculate the roots of $f(r)=0$. This is easily done by means of a power series expansion

$$
r=c_{0} d+c_{1} r_{s}+c_{2} r_{s}^{2}+\cdots
$$

We find

$$
\begin{gathered}
r_{1}=d\left(1-\frac{\delta}{2}-\frac{3}{8} \delta^{2}+O\left(\delta^{3}\right)\right) \\
r_{2}=-d\left(1+\frac{\delta}{2}-\frac{3}{8} \delta^{2}+O\left(\delta^{3}\right)\right) \\
r_{3}=d\left(\delta+\delta^{3}\right),
\end{gathered}
$$

where

$$
\delta=\frac{r_{s}}{d}
$$

and we have ordered the zeros in the same way as in (3.20). Then as in the last subsection we can calculate the direction of the asymptote (3.21) which now is equal to

$$
\varphi_{\infty}=\mu \mathrm{d} F\left(\Phi_{1}, k^{2}\right)
$$

with $\Phi_{1}$ given by (3.23)

$$
\sin ^{2} \Phi_{1}=\frac{1}{2}+\frac{3}{4} \delta+O\left(\delta^{3}\right)
$$

and $k^{2}$ by (3.24)

$$
k^{2}=2 \delta\left(1-\delta+\frac{25}{8} \delta^{2}\right)
$$

and $\mu$ by (3.25)

$$
\mu=\frac{2}{d}\left(1-\frac{\delta}{2}+\frac{9}{8} \delta^{2}\right)
$$

We want to calculate the light deflection in the postEinsteinian approximation. Using again the expansion (3.27) we have

$$
\varphi_{\infty}=2\left(1-\frac{\delta}{2}+\frac{9}{8} \delta^{2}\right)\left[\Phi_{1}+\frac{\delta}{2}(1-\delta)\left(\Phi_{1}-\frac{1}{2} \sin 2 \Phi_{1}\right)\right] .
$$

From (3.34) we obtain

$$
\Phi_{1}=\frac{\pi}{4}+\frac{3}{4} \delta+O\left(\delta^{3}\right)
$$

Then up to $O\left(\delta^{2}\right)$ we find

$$
\varphi_{\infty}=\frac{\pi}{2}+\delta+\left(\frac{3}{4}+\frac{3}{16} \pi\right) \delta^{2} .
$$

The deflection angle is given by

$$
\Delta \varphi=2\left(\varphi_{\infty}-\frac{\pi}{2}\right)=2 \delta+\left(\frac{3}{2}+\frac{3}{8} \pi\right) \delta^{2} .
$$

Instead of the impact parameter $d$ in $\delta$ (3.32) we would like to use the distance of closest approach $r_{1}$
(2.24) in the form

$$
\delta_{1}=\frac{r_{s}}{r_{1}}
$$

The two are related by

$$
\delta=\delta_{1}-\frac{1}{2} \delta_{1}^{2}+O\left(\delta_{1}^{3}\right)
$$

which leads to

$$
\Delta \varphi=2 \delta_{1}+\left(\frac{1}{2}+\frac{3}{8} \pi\right) \delta_{1}^{2} .
$$

The first term $2 \delta_{1}$ is Einstein's result.

\section{The Motion in Time $t=t(\varphi)$}

By dividing (2.7) by (2.10) we find

$$
\begin{aligned}
\frac{\mathrm{d} t}{\mathrm{~d} r} & =E \frac{r}{r-r_{s}}\left[E^{2}-\left(1-\frac{r_{s}}{r}\right)\left(m^{2}+\frac{L^{2}}{r^{2}}\right)\right]^{-1 / 2} \\
& =\frac{E}{L} \frac{r^{3}}{\left(r-r_{s}\right) \sqrt{f(r)}} .
\end{aligned}
$$

We choose $t=0$ at the point of closest approach $r=r_{1}$ and $\mathrm{ge}_{\mathrm{t}}$

$$
t=\frac{E}{L} \int_{r_{1}}^{r} \frac{\mathrm{d} x}{\sqrt{f(x)}}\left(x^{2}+r_{s} x+r_{s}^{2}+\frac{r_{s}^{3}}{x-r_{s}}\right) .
$$

This is a sum of elliptic integrals of first, second and third kind. The coordinate time is given as a function of $r$ by calculating these. However, we want $t$ as a function of $\varphi$ and, therefore, use the substitution (6.7) of the Appendix again

$$
\begin{gathered}
x=r_{1}+\frac{f^{\prime}\left(r_{1}\right)}{4 P(\varphi)-f^{\prime \prime}\left(r_{1}\right) / 6} \\
\frac{\mathrm{d} x}{\sqrt{f(x)}}=\mathrm{d} \varphi,
\end{gathered}
$$

where the last relation follows from (2.11).

The last integral $O\left(r_{s}^{3}\right)$ in (4.2) is a small correction and we neglect it at the moment. Then integrals of the following form remain to be calculated

$$
J_{n}(\varphi)=\int_{0}^{\varphi} \frac{\mathrm{d} u}{(P(u)-P(v))^{n}}
$$

where we have set

$$
P(v)=\frac{f^{\prime \prime}\left(r_{1}\right)}{24} .
$$

Such integrals are known ([7], vol.4, p.109-110) 


$$
\begin{gathered}
J_{1}(\varphi)=\frac{1}{P^{\prime}(v)}\left[2 \zeta(v) \varphi+\log \frac{\sigma(v-\varphi)}{\sigma(v+\varphi)}\right] \\
J_{2}(\varphi)=-\frac{1}{P^{\prime 2}(v)}[\zeta(\varphi+v)+\zeta(\varphi-v) \\
\left.+2 P(v) \varphi+P^{\prime \prime}(v) J_{1}(\varphi)\right] .
\end{gathered}
$$

These results are easily verified by differentiating and using addition formulas. Of course $J_{0}(\varphi)$ is just the polar angle $\varphi$. Then (4.2) leads to the desired result for $t(\varphi)$ :

$$
t(\varphi)=\frac{E}{L}\left\{\tau_{0} \varphi+\tau_{1} J_{1}(\varphi)+\tau_{2} J_{2}(\varphi)\right\}+O\left(r_{s}^{3}\right) .
$$

where

$$
\begin{gathered}
\tau_{0}=r_{1}^{2}+r_{s} r_{1}+r_{s}^{2} \\
\tau_{1}=\left(\frac{r_{1}}{2}+\frac{r_{s}}{4}\right) f^{\prime}\left(r_{1}\right) \\
\tau_{2}=\frac{f^{\prime 2}\left(r_{1}\right)}{16} .
\end{gathered}
$$

Again we evaluate this for bounded orbits in the post-Newtonian approximation by means of the expansion in Theta functions.

The quantity $v$ in $(4.8-11)$ is given as the zero of (3.18). Introducing

$$
V=\frac{\pi}{2 \omega} v
$$

we find

$$
\cos 2 V=-\frac{1}{\varepsilon}-\frac{r_{s}}{r_{1}} \frac{3 \varepsilon+1}{2 \varepsilon} \equiv-\beta .
$$

Since $\varepsilon<1, V$ is complex:

$$
2 V=\pi+i \log \left(\beta+\sqrt{\beta^{2}-1}\right) \equiv \pi+2 i b
$$

Using ([2], eq.18.10.6)

$$
\begin{aligned}
P^{\prime}(v) & =-\frac{\pi^{3}}{4 \omega^{3}} \frac{\vartheta_{2}(V) \vartheta_{3}(V) \vartheta_{4}(V) \vartheta_{1^{\prime}}^{3}(0)}{\vartheta_{2}(0) \vartheta_{3}(0) \vartheta_{4}(0) \vartheta_{1}^{3}(V)} \\
& =-\frac{\pi^{3}}{4 \omega^{3}} \frac{\cos V}{\sin ^{3} V}+O\left(q^{2}\right)
\end{aligned}
$$

we obtain

$$
\begin{aligned}
P^{\prime}(v) & =i \frac{\pi^{3}}{2 \omega^{3}} \frac{\left(\beta^{2}-1\right)^{1 / 2}}{(\beta+1)^{2}}+O\left(r_{s}^{2}\right) \\
& =i \frac{\varepsilon}{2} \frac{\sqrt{1-\varepsilon^{2}}}{(1+\varepsilon)^{2}}\left(1+\frac{r_{s}}{r_{1}} \frac{3 \varepsilon^{2}+4 \varepsilon-5}{1-\varepsilon^{2}}\right) .
\end{aligned}
$$

Similarly we calculate $\zeta(v)$ from ([2], eq.18.10.7)

$$
\zeta(v)=\frac{\eta v}{\omega}+\frac{\pi}{2 \omega} \frac{\vartheta_{1}^{\prime}(V)}{\vartheta_{1}(V)}
$$

where

$$
\eta=\zeta(\omega)=-\frac{\pi^{2}}{12 \omega} \frac{\vartheta_{1}^{\prime \prime \prime}(0)}{\vartheta_{1}^{\prime}(0)}
$$

and $\sigma(z)$ from ([2], eq.18.10.8)

$$
\sigma(z)=\frac{2 \omega}{\pi} \exp \left(\frac{\eta z^{2}}{2 \omega}\right) \frac{\vartheta_{1}(Z)}{\vartheta_{1}^{\prime}(0)}, \quad Z=\frac{\pi z}{2 \omega} .
$$

This implies

$$
\begin{aligned}
\log \frac{\sigma(v-\varphi)}{\sigma(v+\varphi)} & =-2 \frac{\eta}{\omega} v \varphi+\log \frac{\sin (V-\Phi)}{\sin (V+\Phi)}+O\left(q^{2}\right) \\
& =-2 \frac{\eta}{\omega} v \varphi+\log \frac{\cos (i b-\Phi)}{\cos (i b+\Phi)} \\
& \equiv-2 \frac{\eta}{\omega} v \varphi+2 i \alpha(\varphi),
\end{aligned}
$$

where $\alpha$ is given by

$$
\alpha(\varphi)=\arctan (\tan \Phi \tanh b) .
$$

Then $J_{1}(\varphi)$ (4.6) is equal to

$$
J_{1}(\varphi)=-\frac{2 \omega^{2}}{\pi^{2}}(\beta+1) \varphi+\frac{4 \omega^{3}}{\pi^{3}} \frac{(\beta+1)^{2}}{\sqrt{\beta^{2}-1}} \alpha(\varphi)+O\left(\frac{r_{s}}{r_{1}}\right)^{2} .
$$

To expand this in the post-Newtonian order we first calculate $\alpha(\varphi)$ from

$$
\tan \alpha=\sqrt{\frac{1-\varepsilon}{1+\varepsilon}} \tan \Phi+\frac{r_{s}}{r_{1}} \varepsilon \frac{1+3 \varepsilon}{1-\varepsilon^{2}} \sqrt{\frac{1-\varepsilon}{1+\varepsilon}} \tan \Phi .
$$

Introducing

$$
\psi=2 \arctan \left(\sqrt{\frac{1-\varepsilon}{1+\varepsilon}} \tan \Phi\right)
$$

we get

$$
\alpha=\frac{\psi}{2}+\frac{r_{s}}{r_{1}} \frac{\varepsilon}{4} \frac{1+3 \varepsilon}{1-\varepsilon^{2}} \sin \psi .
$$

As before in (3.19) we do not expand $\Phi$ in (4.21). However, if one does so one finds a contribution $O\left(r_{s} / r_{1}\right)$

$$
\psi=2 \arctan \left(\sqrt{\frac{1-\varepsilon}{1+\varepsilon}} \tan (\varphi / 2)\right)-\frac{3}{2} \frac{r_{s}}{r_{1}} \sqrt{\frac{1-\varepsilon}{1+\varepsilon}} \frac{\varphi}{1+\varepsilon \cos \varphi}
$$

where the first term, say $\psi_{N}$, is the parameter which appears in Newtonian mechanics (Kepler's equation, see 
below (4.29)). Now the expansion of $J_{1}(\varphi)$ is given by

$$
\begin{gathered}
J_{1}(\varphi)=J_{1}^{0}(\varphi)+\frac{r_{s}}{r_{1}} J_{1}^{1}(\varphi) \\
J_{1}^{0}(\varphi)=2 \frac{1+\varepsilon}{\varepsilon}\left(\psi \sqrt{\frac{1+\varepsilon}{1-\varepsilon}}-\varphi\right) \\
J_{1}^{1}(\varphi)=\frac{1}{\varepsilon}[-\varphi(7+3 \varepsilon)+\varepsilon) \frac{1+3 \varepsilon}{1-\varepsilon^{2}} \sqrt{\frac{1+\varepsilon}{1-\varepsilon}} \sin \psi \\
\left.+2 \psi \sqrt{\frac{1+\varepsilon}{1-\varepsilon}} \frac{5-4 \varepsilon-3 \varepsilon^{2}}{1-\varepsilon}\right] .
\end{gathered}
$$

For $\varepsilon \rightarrow 0$ we have the simple finite limit

$$
\begin{gathered}
\left.J_{1}(\varphi)\right|_{\varepsilon=0}=\frac{2 \omega}{\pi}\left(1+3 \frac{r_{s}}{r_{1}}\right)(2 \Phi-\sin 2 \Phi) \quad \text { This finally leads to } \\
J_{2}^{0}(\varphi)=\frac{4}{\varepsilon} \frac{(1+\varepsilon)^{2}}{1-\varepsilon}\left[\varphi-\frac{(1+\varepsilon) \sin 2 \Phi}{1+\varepsilon \cos 2 \Phi}+\frac{2 \varepsilon)-1}{2(1+\varepsilon)} J_{1}^{0}(\varphi)\right] \\
J_{2}^{1}(\varphi)=\frac{2}{\varepsilon} \frac{(1+\varepsilon)^{2}}{1-\varepsilon}\left[\varphi \frac{15-12 \varepsilon-11 \varepsilon^{2}}{1-\varepsilon^{2}}-\frac{\sin 2 \Phi}{1+\varepsilon \cos 2 \Phi}\left(\frac{1+4 \varepsilon+3 \varepsilon^{2}}{1+\varepsilon \cos 2 \Phi}+\frac{17-13 \varepsilon-12 \varepsilon^{2}}{1-\varepsilon}\right)\right. \\
\left.+J_{1}^{0}(\varphi) \frac{6-15 \varepsilon+8 \varepsilon^{2}-\varepsilon^{3}}{(1+\varepsilon)^{2}(1-\varepsilon)}-J_{1}^{1}(\varphi) \frac{1-2 \varepsilon}{1+\varepsilon}\right] .
\end{gathered}
$$

Again we do not expand $\Phi$ (3.16) in order to keep the perihelion precession as precise as possible. The limit for $\varepsilon \rightarrow 0$ is equal to

$\left.J_{2}(\varphi)\right|_{\varepsilon=0}=2 \frac{\omega}{\pi}\left(1+6 \frac{r_{s}}{r_{1}}\right)(6 \Phi-4 \sin 2 \Phi+\sin 2 \Phi \cos 2 \Phi)$.

In the final result (4.8) for the time

$$
t(\varphi)=t_{0}(\varphi)+\frac{r_{s}}{r_{1}} t_{1}(\varphi)
$$

the pre-factor $E / L$ also gives a correction:

$$
\begin{aligned}
& \frac{E}{L}=\sqrt{\frac{2}{r_{s} r_{1}(1+\varepsilon)}}\left(1-\frac{r_{s}}{r_{1}(1+\varepsilon)}\right) \\
& t_{1}(\varphi)=t_{0}(\varphi) \frac{\varepsilon}{1+\varepsilon}+\varepsilon \sqrt{\frac{2 r_{1}^{3}}{r_{s}(1+\varepsilon)}}\left[\frac{1}{1+\varepsilon} J_{1}^{1}(\varphi)-\frac{1}{2} \frac{\varepsilon+5}{(1+\varepsilon)^{2}} J_{1}^{0}(\varphi)+\frac{\varepsilon}{4\left(1+\varepsilon^{2}\right)} J_{2}^{1}(\varphi)-\frac{\varepsilon}{2} \frac{3+\varepsilon}{(1+\varepsilon)^{3}} J_{2}^{0}(\varphi)\right] .
\end{aligned}
$$

As in (3.19) the post-Newtonian correction vanishes for circular motion $\varepsilon=0$.

\section{Gravitational Red Shift}

The study of Schwarzschild geodesics is relevant for the investigation of the recently discovered S-stars near the which follows from (2.19-21). In $t_{0}$ the terms proportional to $\varphi$ cancel

$$
t_{0}(\varphi)=\sqrt{\frac{2 r_{1}^{3}}{r_{s}(1+\varepsilon)}} \frac{1+\varepsilon}{1-\varepsilon}\left(\frac{\psi}{\sqrt{1-\varepsilon^{2}}}-\frac{\varepsilon \sin 2 \Phi}{1+\varepsilon \cos 2 \Phi}\right) .
$$

Approximating $2 \Phi$ by $\varphi$ this is in agreement with Kepler's equation

$$
t_{0}(\varphi)=\sqrt{\frac{2 r_{1}^{3}}{r_{s}(1-\varepsilon)^{3}}}(\psi-\varepsilon \sin \psi) .
$$

Galactic Center ([1] and references given there). These stars move in the strong gravitational field of the central black hole so that general relativistic effects are observable and the Schwarzschild metric $g_{\mu \nu}$ is a fairly good description of the situation. The measurable quantity of interest is the red shift of spectral lines in the light emitted by the moving star. Therefore we finally consider 
this.

Let $v_{1}$ be the frequency of a given atomic line from the star and $v_{0}$ the frequency of the same line observed in the rest frame of the galaxy. If $\mathrm{d} x^{\mu} / \mathrm{d} t$ is the velocity of the star, the two frequencies are related by ([9] p.83, equ. 3.5.6)

$$
\frac{v_{1}}{v_{0}}=\frac{\left(g_{\alpha \beta}(x) \frac{\mathrm{d} x^{\alpha}}{\mathrm{d} t} \frac{\mathrm{d} x^{\beta}}{\mathrm{d} t}\right)^{1 / 2}}{g_{00}(X)^{1 / 2}} .
$$

We assume that the observer at $X$ is far away from the center such that the denominator can be approximated by 1. For a star moving in the plane $\vartheta=\pi / 2$ we have

$$
g_{\alpha \beta}(x) \frac{\mathrm{d} x^{\alpha}}{\mathrm{d} t} \frac{\mathrm{d} x^{\beta}}{\mathrm{d} t}=e^{v}-e^{v}\left(\frac{\mathrm{d} r}{\mathrm{~d} t}\right)^{2}-r^{2}\left(\frac{\mathrm{d} \varphi}{\mathrm{d} t}\right)^{2} .
$$

From (2.10) and (2.7) we find

$$
\frac{\mathrm{d} r}{\mathrm{~d} t}=e^{v} \sqrt{1-\frac{e^{v}}{E^{2}}\left(m^{2}+\frac{L^{2}}{r^{2}}\right)}
$$

and (2.8) gives

$$
\frac{\mathrm{d} \varphi}{\mathrm{d} t}=\frac{L}{E} \frac{e^{v}}{r^{2}} .
$$

Substituting all this into (5.1) we see that $L$ drops out and we end up with the simple result

$$
\frac{v_{1}}{v_{0}}=\frac{m}{E} e^{v}=\frac{m}{E}\left(1-\frac{r_{s}}{r(\varphi)}\right) .
$$

By (2.20) we can express $E$ by the perihelion $r_{1}$ and aphelion $r_{2}$

$$
E^{2}=m^{2}\left(1-\frac{r_{s}}{r_{1}+r_{2}+r_{3}}\right)
$$

where $r_{3}$ is the small correction (2.21). Then we finally get

$$
\frac{v_{1}}{v_{0}}=\left(1-\frac{r_{s}}{r(\varphi)}\right)\left(1-\frac{r_{s}}{r_{1}+r_{2}+r_{3}}\right)^{-1 / 2} .
$$

The lowest order $O\left(r_{s}\right)$ is equal to

$$
\frac{v_{1}}{v_{0}}=1-\frac{r_{s}}{r(\varphi)}+\frac{r_{s}}{2\left(r_{1}+r_{2}\right)}+O\left(r_{s}^{2}\right) \text {. }
$$

Since the last term is always smaller than the second one we indeed have red shift $\lambda_{1}>\lambda_{0}$. Of course, it is maximal at the perihelion where $r=r_{1}$ is minimal. The total observed red shift is obtained by multiplying (5.5) with the Doppler factor $\left(1+v_{r}\right)^{-1}$ where $v_{r}$ is the component of the relative velocity along the direction from the observer to the star ([9], p.30).

\section{Acknowledgments}

It is a pleasure to acknowledge elucidating discussions with Prasenjit Saha, in particular the introduction into the fascinating field of Galactic-center stars. I also thank Raymond Angélil for showing his simulations of the corresponding dynamics.

\section{References}

[1] D. J. D’Orazio and P. Saha, "An Analytic Solution for Weak-field Schwarzschild Geodesics," Monthly Notices of the Royal Astronomical Society, Vol. 406, pp. 2787-2792.

[2] M. Abramowitz and I. A. Stegun, "Handbook of Mathematical Functions," Dover Publications, Inc., New York.

[3] G. Scharf and Gen. Relativ. Gravit, "From Massive Gravity to Modified General Relativity," General Relativity and Gravitation, Vol. 42, pp. 471-487. doi: 10.1007/s10714-009-0864-0

[4] S. Chandrasekhar, "The Mathematical Theory of Black Holes," Oxford/New York, Clarendon Press/Oxford University Press, 1983.

[5] E. T. Whittaker and G. N. Watson, "A Course of Modern Analysis," Cambridge University Press, 1950.

[6] A. Erdelyi et al., "Higher Transcendental Functions," McGraw-Hill Book Co., Inc., New York, 1953.

[7] J. Tannery, J. Molk, "Fonctions elliptiques," Chelsea Publishing Company, Bronx, New York, 1972.

[8] Ch. Darwin, Proc. Roy. S. London A 249 (1959) 180, A 263 (1961) 39.

[9] S. Weinberg, "Gravitation and Cosmology," John Wiley, New York, 1972.

[10] G. Scharf, "Quantum Gauge Theories-Spin One and Two," Google-Books (2010) free access.

[11] Y. Hagihara, Japanese J. Astron. Geophys. 8 (1930) 68. 


\section{Appendix: Integration of the Differential} Equation

We closely follow Whittaker and Watson ([5], p.452). With the notation of the paper (2.11), let

$$
\varphi=\int_{r_{1}}^{r} \frac{\mathrm{d} x}{\sqrt{f(x)}}
$$

where $r_{1}$ is any zero, $f\left(r_{1}\right)=0$. By Taylor's theorem, we have

$$
\begin{aligned}
f(x)= & 4 A_{3}\left(x-r_{1}\right)+6 A_{2}\left(x-r_{1}\right)^{2} \\
& +4 A_{1}\left(x-r_{1}\right)^{3}+A_{0}\left(x-r_{1}\right)^{4},
\end{aligned}
$$

where

$$
\begin{gathered}
A_{0}=a_{0}, \quad A_{1}=a_{0} r_{1}+a_{1} \\
A_{2}=a_{o} r_{1}^{2}+2 a_{1} r_{1}+a_{2}, \\
A_{3}=a_{0} r_{1}^{3}+3 a_{1} r_{1}^{2}+3 a_{2} r_{1}+a_{3} .
\end{gathered}
$$

Introducing the new integration variable

$$
s=\left(x-r_{1}\right)^{-1}, \quad s_{1}=\left(r-r_{1}\right)^{-1},
$$

we have

$$
\varphi=\int_{s_{1}}^{\infty}\left[4 A_{3} s^{3}+6 A_{2} s^{2}+4 A_{1} s+A_{0}\right]^{-1 / 2} \mathrm{~d} s .
$$

To remove the second term in the cubic we set

$$
s=\frac{1}{A_{3}}\left(z-\frac{A_{2}}{2}\right), \quad s_{1}=\frac{1}{A_{3}}\left(z_{1}-\frac{A_{2}}{2}\right)
$$

and we get

$$
\begin{aligned}
\varphi= & \int_{z_{1}}^{\infty}\left[4 z^{3}-\left(3 A_{2}^{2}-4 A_{1} A_{3}\right) z\right. \\
& \left.-\left(2 A_{1} A_{2} A_{3}-A_{2}^{3}-A_{0} A_{3}^{2}\right)\right]^{-1 / 2} \mathrm{~d} x
\end{aligned}
$$

The coefficients of $z$ and $z^{0}$ are just the invariants $g_{2}, g_{3}$ (2.14-15) of the original quartic.

Now the inversion of the integral gives Weierstrass' $P$-function

$$
z_{1}=P\left(\varphi ; g_{2}, g_{3}\right) .
$$

From (6.2) and (6.3) we have

$$
r=r_{1}+\frac{A_{3}}{z_{1}-A_{2} / 2}
$$

and hence

$$
r=r_{1}+\frac{f^{\prime}\left(r_{1}\right)}{4 P(\varphi)-f^{\prime \prime}\left(r_{1}\right) / 6} .
$$

